\title{
Statistical models - causal relationship and confounding
}

Ranstam J, Cook JA

Randomised trials are designed to eliminate systematic error (bias) in the estimate of the effect of an intervention (usually a treatment) through randomised allocation of patients to treatment the intervention with concealment of allocation. In an observational study, no such intervening in the treatment is made and the outcome is merely "observed" along with other relevant information (e.g. pre-operative characteristic of patients). Unless the groups of individuals who receive the intervention and control are similar (or such groups can be created), selection, information and confounding bias will need to be addressed in the statistical analysis.

The most commonly used method to adjust a parameter estimate (e.g. the beneficial effect of a treatment, or the risk of exposure to a hazardous agent) is to develop a statistical model that includes variables representing not only exposure (e.g. intervention or control) and outcome but also the potential confounding factors to adjust for.

Data-driven methods are often used to select confounding variables. Screening of candidate variables typically based upon univariate analyses, using forward or backward stepwise multiple regression, and model development based upon a measure which assesses relative model performance (for example using Akaike's information criterion) are commonly used. However, confounding bias may undermine these process as the causal relations between variables need to be taken into account, something which cannot be achieved by blindly apply the methods.

Four simple examples of causal relationships are shown in Figure 1 which involves an exposure (E), an outcome (O), and a third factor (C). The cause-effect relationships between variables (including direction) are shown as arrows. Depending these links the consequences of including $\mathrm{C}$ in a statistical adjustment model will differ. First, if the third factor is independent of the exposure or the outcome or both of these $\left(\mathrm{C}_{0}\right)$, then adjustment is unnecessary (in terms of bias). It will reduce the degrees of freedom though this is not problematic in most studies. Second, a classic confounder $\left(\mathrm{C}_{1}\right)$ is an independent risk factor that has a systematically imbalanced distribution in the studied exposure groups. An unadjusted estimate of the effect of $\mathrm{E}$ on $\mathrm{O}$, ignoring the relations with $\mathrm{C}_{1}$, is likely to be a biased estimate. The remedy is to include $\mathrm{C}_{1}$ in the statistical model, which, in principle, will provide an unbiased effect estimate. A factor on the pathway between exposure and effect $\left(\mathrm{C}_{2}\right)$ is, however, not a confounding factor and should not be adjusted for in this manner. If $\mathrm{C}_{2}$ nevertheless is included in the statistical model, it generates (overadjustment) bias (1).Third, a factor that is affected by both exposure and outcome $\left(\mathrm{C}_{3}\right)$ is called a collider. Including a collider in the statistical model generates collider-stratification (also known as endogeneous selection) bias. Give the potential complexity involved with multiple variables a graphical method is often used facilitate consideration of relationships and understand the implications of a specific analysis(3).

Another, increasingly popular, method to adjust for confounding bias is the propensity score approach. This seeks to addresses the imbalance of potential confounding factors between the observed groups rather than the influence of factors on the outcome, a fundamentally different method (4). In brief, it consists in first fitting one statistical model to estimate each patient's propensity (probability) of being exposed. Second, these propensity scores can then 
be used either to create similar groups or to adjusted for in the model.

It is worth highlighting that the (causal) relationship between included variables, and the process by which adjustment variables are selected, has an impact upon the validity of the propensity score method $(5,6)$. 


\section{References}

1. Schisterman EF, Cole SR, Platt RW. Overadjustment bias and unnecessary adjustment in epidemiological studies. Epidemiol 2009;20:488-495.

2. Greenland S. Quantifying biases in causal models: classical confounding vs colliderstratification bias. Epidemiology 2003; 14:300-306.

3. Shrier I, Platt RW. Reducing bias through directed acyclic graphs. BMC Med Res Methodol 2008;8:70.

4. Adamina M, Guller U, Weber WP, Oertli D. Propensity scores and the surgeon. BJS 2006;93:389-394.

5. Brookhart MA, Schneeweiss S, Rothman KJ, Glynn RJ, Avorn J, Stürmer T. Variable Selection for Propensity Score Models. Am J Epidemiol 2006;163:1149-1156.

6. Sjölander A. Propensity scores and M-structures. Stat Med 2009;28:1416-1420.

\section{Word count 585}


Figure 1. Confounding bias and causal relation between variables

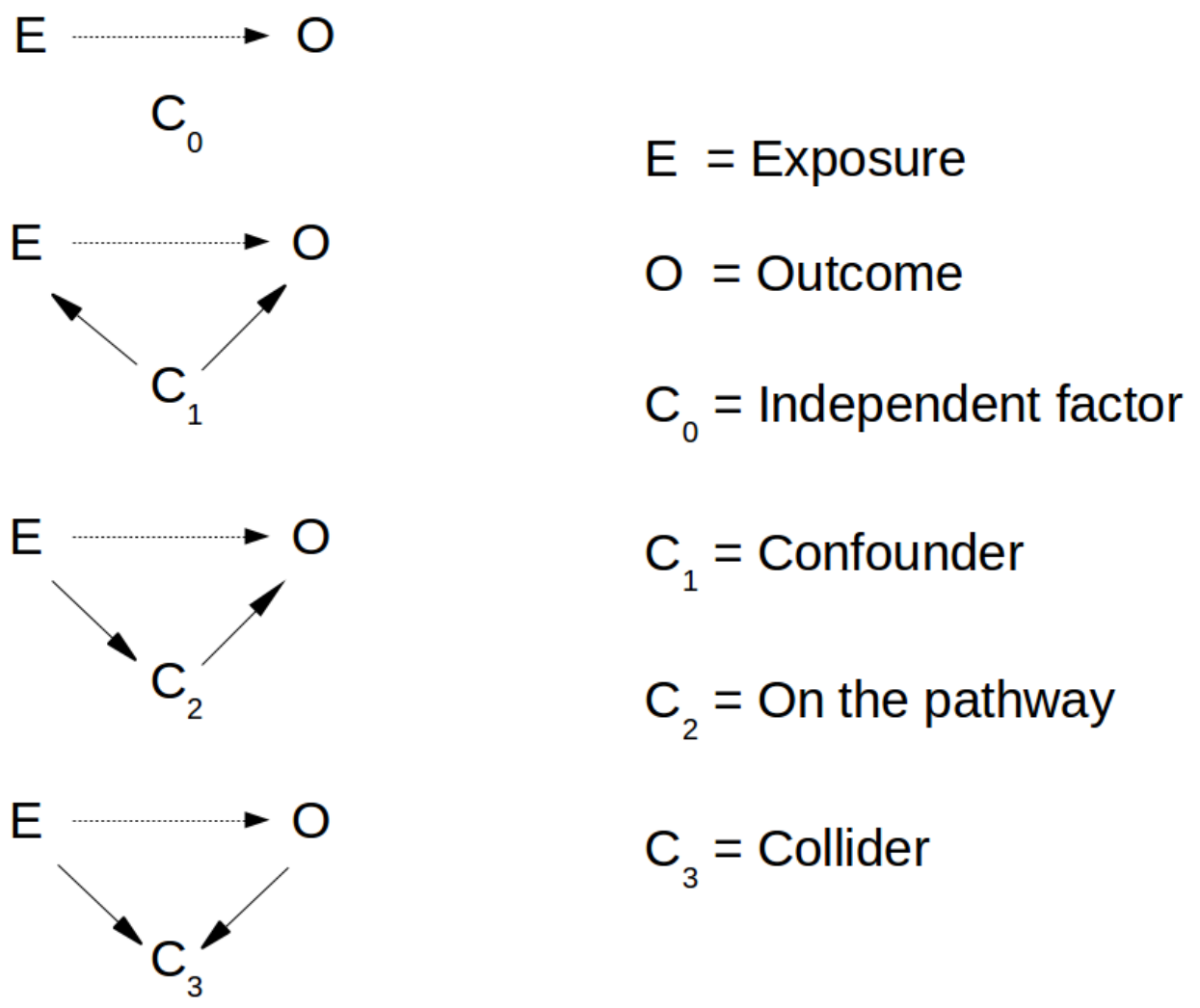

